\title{
Efeitos da fragmentação florestal na bacia do alto curso do rio Gramame, município de Pedras de Fogo (PB)
}

\author{
Effects of forest fragmentation in the basin of the upper course of the river \\ Gramame, Pedras de Fogo county (PB)
}

SILVA $^{1}$, J. B.;VITAL ${ }^{2}$, S. R. O.; CHAVES ${ }^{3}$, J. R.

josivan_bernardo@hotmail.com

\begin{abstract}
Resumo
As espécies são sensíveis às mudanças físicas do ambiente e necessitam de condições adequadas para garantir sua sobrevivência. As florestas apresentam um grande mosaico de condições com vários microclimas. A floresta Atlântica, especificamente, se destaca, no mundo, por apresentar altos índices de biodiversidade, e também, várias espécies endêmicas. No município de Pedras de Fogo (PB), esse bioma se apresenta muito fragmentado, o que foi constatado a partir de análises feitas por meio de técnicas de geoprocessamento, evidenciando a existência de 35 fragmentos com formas e tamanhos variados. A maior parte destes apresentam formas irregulares e áreas núcleos bastante reduzidas, tendo como consequência, o desenvolvimento de impactos negativos sobre as espécies, que respondem de formas variadas às perturbações. Além disso, foram avaliadas as distâncias entre os fragmentos, revelando um baixo grau de isolamento, principalmente na porção oeste da bacia. Desse modo, entende-se que é preciso aprofundar os estudos nessa área, com um possível levantamento das espécies e uma coleta de dados mais apurada sobre as reais condições físicas no interior dos fragmentos.
\end{abstract}

Palavras-chave: Fragmentação florestal, Floresta Atlântica, bacia do alto curso do rio Gramame.

\begin{abstract}
The species are sensitive to the physical environment changes and require appropriate conditions to ensure their survival. Forests have a large mosaic of conditions with various microclimates. The Atlantic forest, in particular, stands out in the world, due to its high levels of biodiversity, and also several endemic species. In the municipality of Pedras de Fogo (PB), this biome appears very fragmented, which was found from analysis by means of geoprocessing techniques, showing the existence of 35 fragments with varying shapes and sizes. Most of these have irregular shapes and very small nuclei areas, resulting in the development of negative impacts on the species, that respond in various ways to disturbances. In addition, the distances between the fragments were assessed, revealing a low-grade insulation, particularly in the western portion of the basin. Thus, it is understood that it is necessary to further study in this area, with a possible survey of the species and more accurate data collection on the actual physical conditions within the fragments.
\end{abstract}

Keywords: Forest fragmentation, Atlantic Forest, basin of the upper course of the river Gramame.

\section{INTRODUÇÃO}

As espécies necessitam de um complexo de condições favoráveis à sua sobrevivência. Essas condições propiciarão um sucesso reprodutivo e evolutivo que garantirá a continuidade de seus descendentes (ODUM, 2004).

As florestas apresentam um mosaico de condições que possibilitam a existência de uma diversidade de seres que dependem dela como fonte direta ou indireta de energia. Além disso, elas possibilitam a formação de microclimas que atuam sobre os seres que ali habitam (BRASIL, 2003).

A floresta atlântica brasileira se destaca por apresentar índices elevados de biodiversidade, os maiores já encontrados em florestas tropicais, como também por apresentar altas taxas de endemismo (PEREIRA; ALVES, 2007).

${ }^{1}$ Josivan Bernardoda Silva, Departamento de Sistemática e Ecologia, Laboratório de Herpetologia, Universidade Federal da Paraíba, João Pessoa/PB, Brasil.

${ }^{2}$ Saulo Roberto de Oliveira Vital, Departamento de Geografia, Laboratório de Geoprocessamento e Geografia Física, Universidade Federal do Rio Grande do Norte, Caicó/RN, Brasil.

3 Jailson Rodrigues Chaves, Faculdade de Ciência e Tecnologia Professor Dirson Marciel de Barros, PE, Brasil. 
O município de Pedras de Fogo, localizado no estado da Paraíba, tem sua economia movimentada principalmente pela agricultura, onde é predominante o cultivo de cana-de-açúcar, que tomou o espaço antes ocupado pela floresta atlântica (LIMA, et al., 2011). Esse tipo de cultura vem modificando a paisagem e alterando o comportamento de espécies que passaram a ocupar pequenos fragmentos. Levando em consideração esta problemática, o presente trabalho visa analisar os efeitos da fragmentação sobre a Floresta Atlântica no município de Pedras de Fogo e suas possíveis consequências para as espécies animais e vegetais. Para tanto, serão utilizadas técnicas de geoprocessamento, sensoriamento remoto e métricas de paisagem.

A bacia do alto curso do rio Gramame encontra-se localizada no município de Pedras de Fogo, situado na microrregião de Pedras de Fogo e na mesorregião da mata paraibana (Figura 1). A bacia analisada apresenta uma área de $54 \mathrm{~km}^{2}$, estando localizada numa região cuja altitude alcança o valor médio de 70 metros. O acesso é feito a partir de João Pessoa, pelas rodovias BR-230 e PB030 (BRASIL, 2005).

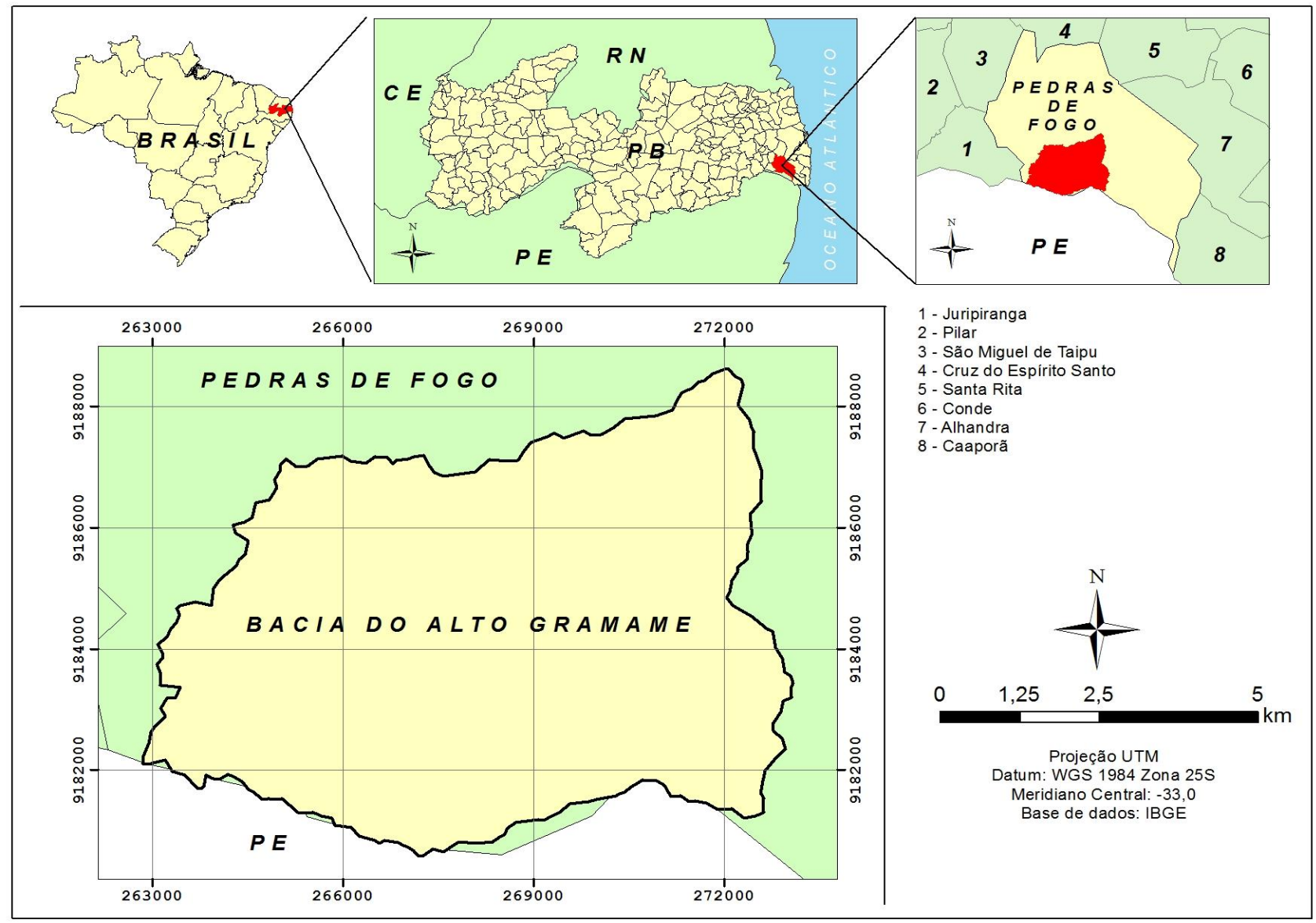

Figura 1. Localização da bacia do alto curso do rio Gramame.

A vegetação é composta, em sua maioria, por remanescentes de Floresta Atlântica, onde se observa uma cobertura heterogênea, com indivíduos de 5,0 a 10,0 metros de altura, tendo espécies pertencentes principalmente as famílias: Anacardiaceae, Boraginaceae, Fabaceae e Rubiaceae. No 
entanto, em virtude da urbanização, este domínio já se encontra bastante alterado, onde também se observa espécies secundárias, tais como: Cecropia sp., Miconia albicans Triana e a Miconia calvensis DC. (PEREIRA; ALVES, 2007). Além disso, também estão presentes espécies de Manguezal, com forte ocorrência nos estuários do rio Paraíba do Norte e Gramame, além de pequenas manchas de Cerrado no topo dos tabuleiros costeiros (MELO et al., 2001).

No tocante à hidrografia, observa-se que o município de Pedras de Fogo está inserido nas bacias dos rios Gramame e Paraíba, na região do baixo Paraíba. Seus principais afluentes são os rios Gramame, Mamuaba, Mumbaba, Utinga, Pau-Brasil e Papocas (BRASIL, 2005). É importante ressaltar que ao norte de Pedras de Fogo localiza-se a nascente do rio Gramame, tendo como principais afluentes os rios Mumbaba e Mamuaba, que juntos chegam a somar uma área de $580 \mathrm{~km}^{2}$ (BARBOSA; WATANABE; PAZ, 2009).

\section{METODOLOGIA}

Neste trabalho, foram utilizadas técnicas de geoprocessamento para avaliar a quantidade, o tamanho, a distância para o vizinho mais próximo, a relação perímetro/área e a área núcleo dos fragmentos florestais. Um dos dados de grande relevância na análise de fragmentos florestais é a relação perímetro/área (IP), onde o perímetro é dado em metros e a área em metros quadrados. Os resultados indicam as reais condições dos fragmentos referentes à forma dos mesmos. Valores próximos de 1 indicam que os fragmentos apresentam formas mais regulares enquanto os valores próximos de 0 indicam formas bastante irregulares distantes de um círculo perfeito (GUARIZ; CAMPANHARO; PICOLI, 2011).

Outro ponto importante no estudo da paisagem fragmentada é análise das distâncias entre os fragmentos. Essas refletem a qualidade de uma determinada área fragmentada, pois quanto maior a distância, maior o grau de isolamento, o que dificulta a dispersão das espécies, como também torna os fragmentos mais propícios à extinção.

Segundo Almeida (2008), a distância entre um fragmento e o seu vizinho mais próximo pode caracterizar um grau de isolamento baixo (até 60 metros), médio (de 60 a 120 metros), alto (de 120 a 200 metros) e muito alto (superior a 200 metros).

Para a delimitação da área em estudo, que corresponde à bacia do alto curso do rio Gramame, foram utilizados dados do SRTM (Shuttle Radar Topographic Mission), obtidos na plataforma TOPODATA (Banco de Dados Geomorfométricos do Brasil) do INPE (Instituto Nacional de Pesquisas Espaciais) através do endereço: http://www.dsr.inpe.br/topodata/.

O processamento da imagem SRTM foi realizado com o intuito de obter informações de direção e fluxo dos canais, visando a delimitação automática da bacia hidrográfica. A extração dos 
limites da bacia foi validada a partir da análise de seus divisores topográficos com auxílio nas curvas de nível do terreno existentes em cartas topográficas na escala 1:10.000, onde foi constatada a coerência. Os fragmentos foram delimitados com base em imagens de satélite de alta resolução, por meio de técnicas de fotointerpretação e vetorização. Com os fragmentos delimitados, foram obtidos os valores de métrica da paisagem utilizando a função Patch Analyst do ArcGIS 10.3, adquirido a partir de uma licença experimental de 30 dias.

Ao final do procedimento de identificação dos dados, os mesmos foram analisados através de estatística descritiva, sendo armazenados em uma planilha do Microsoft Excel para posterior representação em gráficos e tabelas.

\section{RESULTADOS E DISCUSSÃO}

Análises realizadas na área em estudo através de técnicas de geoprocessamento e sensoriamento remoto mostraram a existência de 35 fragmentos com tamanhos e formas muito variadas. Com a análise dos dados, foi possível reunir os trinta e cinco fragmentos em dois grupos, levando em consideração a classificação de Almeida (2008).

Observando os dados da tabela 1, é possível detectar a predominância de fragmentos pequenos, pois representam $80 \%$ do total existente na bacia. Vale salientar que dentre os fragmentos considerados pequenos 71,4\% apresentam uma área menor que 2 hectares. Isso reforça o que diz Pereira e Alves (2007), quando afirma que a Floresta Atlântica na Paraíba se encontra muito fragmentada.

Tabela 1. Quantidades, áreas e índices perimetrais dos fragmentos da bacia do alto curso do Rio Gramame.

\begin{tabular}{c|c|c|c|c|c|l}
$\begin{array}{c}\text { Número do } \\
\text { Fragmento }\end{array}$ & Área (ha) & $\begin{array}{c}\text { \% da } \\
\text { Área }\end{array}$ & $\begin{array}{c}\text { Área } \\
\text { Núcleo }\end{array}$ & $\begin{array}{c}\text { \% da Área } \\
\text { Núcleo }\end{array}$ & $\begin{array}{c}\text { Índice } \\
\text { Perimetral }\end{array}$ & $\begin{array}{c}\text { Classificação } \\
\text { (ALMEIDA, 2008) }\end{array}$ \\
\hline 1 & 2,84 & 1,79 & 0,06 & 0,13 & 0,03 & Pequeno \\
2 & 1,05 & 0,66 & - & - & 0,04 & Pequeno \\
3 & 5,12 & 3,22 & 1,12 & 2,58 & 0,02 & Médio \\
4 & 1,38 & 0,87 & - & - & 0,04 & Pequeno \\
5 & 3,60 & 2,26 & 0,23 & 0,53 & 0,03 & Pequeno \\
6 & 0,41 & 0,25 & - & - & 0,07 & Pequeno \\
7 & 2,55 & 1,60 & 0,04 & 0,10 & 0,03 & Pequeno \\
8 & 13,80 & 8,68 & 6,55 & 15,17 & 0,01 & Médio \\
9 & 4,21 & 2,65 & 0,65 & 1,52 & 0,03 & Pequeno \\
10 & 0,91 & 0,57 & - & - & 0,04 & Pequeno \\
11 & 1,44 & 0,90 & - & - & 0,04 & Pequeno \\
12 & 15,16 & 9,54 & 4,26 & 9,85 & 0,02 & Médio \\
13 & 2,75 & 1,73 & 0,35 & 0,82 & 0,02 & Pequeno \\
14 & 0,50 & 0,31 & - & - & 0,07 & Pequeno \\
15 & 27,33 & 17,19 & 11,39 & 26,37 & 0,02 & Médio \\
16 & 1,38 & 0,87 & - & - & 0,04 & Pequeno \\
17 & 1,57 & 0,99 & 0,00 & 0,00 & 0,05 & Pequeno \\
18 & 1,08 & 0,68 & - & - & 0,05 & Pequeno
\end{tabular}




\begin{tabular}{l|c|c|c|c|c|l}
19 & 7,60 & 4,78 & 0,01 & 0,03 & 0,03 & Médio \\
20 & 1,15 & 0,73 & - & - & 0,04 & Pequeno \\
21 & 0,70 & 0,44 & - & - & 0,06 & Pequeno \\
22 & 0,79 & 0,50 & - & - & 0,06 & Pequeno \\
23 & 0,13 & 0,08 & - & - & 0,12 & Pequeno \\
24 & 1,06 & 0,67 & - & - & 0,08 & Pequeno \\
25 & 0,33 & 0,21 & - & - & 0,08 & Pequeno \\
26 & 4,89 & 3,08 & 0,19 & 0,45 & 0,03 & Pequeno \\
27 & 2,53 & 1,59 & 0,06 & 0,14 & 0,03 & Pequeno \\
28 & 4,42 & 2,78 & 0,52 & 1,21 & 0,03 & Pequeno \\
29 & 1,85 & 1,17 & - & - & 0,05 & Pequeno \\
30 & 7,78 & 4,89 & 2,30 & 5,33 & 0,02 & Médio \\
31 & 33,88 & 21,31 & 15,44 & 35,75 & 0,01 & Médio \\
32 & 0,99 & 0,62 & - & - & 0,05 & Pequeno \\
33 & 0,65 & 0,41 & - & - & 0,07 & Pequeno \\
34 & 1,35 & 0,85 & 0,02 & 0,04 & 0,03 & Pequeno \\
35 & 1,83 & 1,15 & 0,00 & 0,00 & 0,04 & Pequeno \\
\hline Total & 159,03 & 100 & 43,20 & 100 & - & \\
\hline
\end{tabular}

Fonte: Dados da Pesquisa.

Em relação ao tamanho dos fragmentos, Silva e Souza (2014) afirmam que, quanto menor a área de um fragmento, maior será o efeito e pressões sobre a borda dos mesmos. Esse efeito modifica as condições e possibilita a ocupação de espécies invasoras. Isso traz prejuízos às espécies da área em estudo, tendo em vista o que diz Torezan (2003), quando assegura que uma das implicações do efeito de borda é a alteração do microclima dentro dos fragmentos, podendo ocorrer elevação da temperatura interna.

Levando em consideração o que diz Odum (2004), a temperatura é de grande importância, sendo, na maioria dos casos, um fator limitante, pois junto com a luz, a umidade e as marés controlam as atividades das plantas e animais. As modificações das condições físicas alteram a fisiologia dos organismos e com isso determinam se o ambiente é adequado para a espécie (TOWNSEND; BEGON; HARPER, 2010). Ao analisar que mais de $70 \%$ dos fragmentos da bacia tem área inferior a 2 hectares, pode-se inferir que muito dessas alterações de fato estão acontecendo.

Os dados referentes à relação perímetro/área comprovam o alto grau de fragmentação florestal na área da bacia, pois, para essa variável, os fragmentos tiveram valores muito próximos de zero, revelando que os mesmos apresentam formas muito irregulares, sendo esses menos recomendados para preservação segundo Souza e Silva (2014). Segundo Almeida (2008) a forma do fragmento está diretamente relacionada aos efeitos de borda, mostrando que, quanto menor o valor da relação perímetro/área, maiores serão esses efeitos.

Ao relacionar o índice perimetral com o tamanho dos fragmentos foi possível constatar que os fragmentos menores têm índices perimetrais mais próximos de um, enquanto os maiores mostram uma queda para essa variável (Figura 2). 


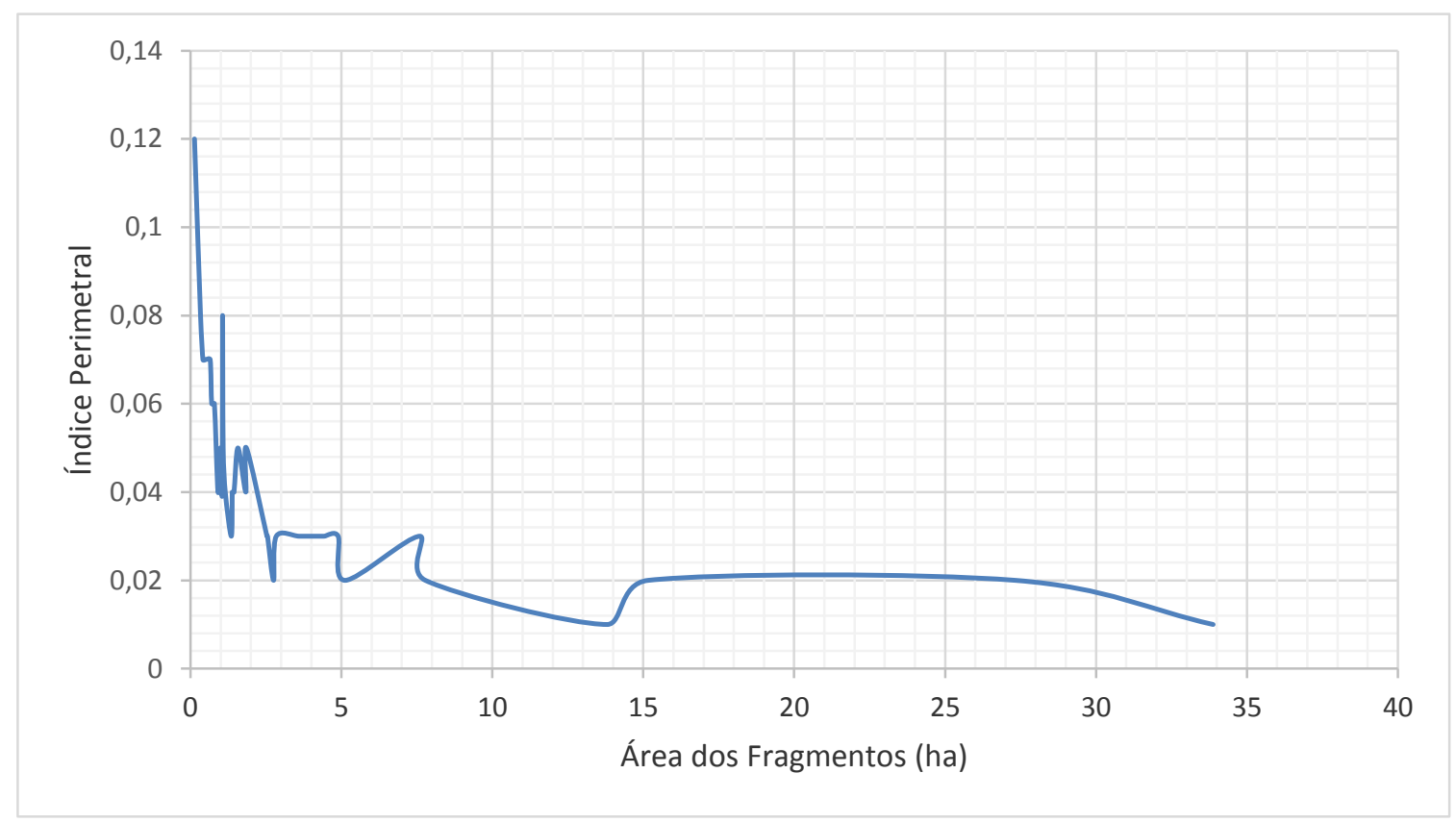

Figura 2. Relação do índice perimetral com a área dos fragmentos em hectares.

Também foram avaliadas as áreas núcleo dos fragmentos, onde se considerou como borda uma distância de 50 metros a partir do perímetro para o interior do fragmento. Os resultados da pesquisa mostraram que devido às formas irregulares e as pequenas dimensões dos fragmentos, a áreas núcleo encontram-se bastante reduzidas, onde 48,5\% não apresentam valores para essa variável e 66,6\% mostraram área núcleo muito pequenas com valores abaixo de 1,0 ha. Esses resultados estão diretamente relacionados com o cultivo de cana-de-açúcar, que predomina na região e vem tomando o espaço da vegetação nativa ao longo dos anos.

A redução das áreas florestais diminui a qualidade dentro dos fragmentos, pois quanto menor o tamanho do mesmo, maior será a exposição daquele habitat aos efeitos de borda. Em um estudo realizado com um fragmento depois que sua área circundante foi retirada para dar lugar a atividades pecuárias em Manaus, foi constatado que a mortalidade das árvores no exterior do fragmento foi várias vezes maior que as que se encontravam no interior do mesmo (RICKLEFS, 2010). Isso mostra a vulnerabilidade da vegetação na borda dos fragmentos como também reforça a ideia da modificação do microclima dentro dos fragmentos que, por sua vez, afeta as populações animais residentes.

Os dados sobre o grau de isolamento na área em estudo, revelam a predominância de fragmentos com baixo e muito alto isolamento (Figura 3). 


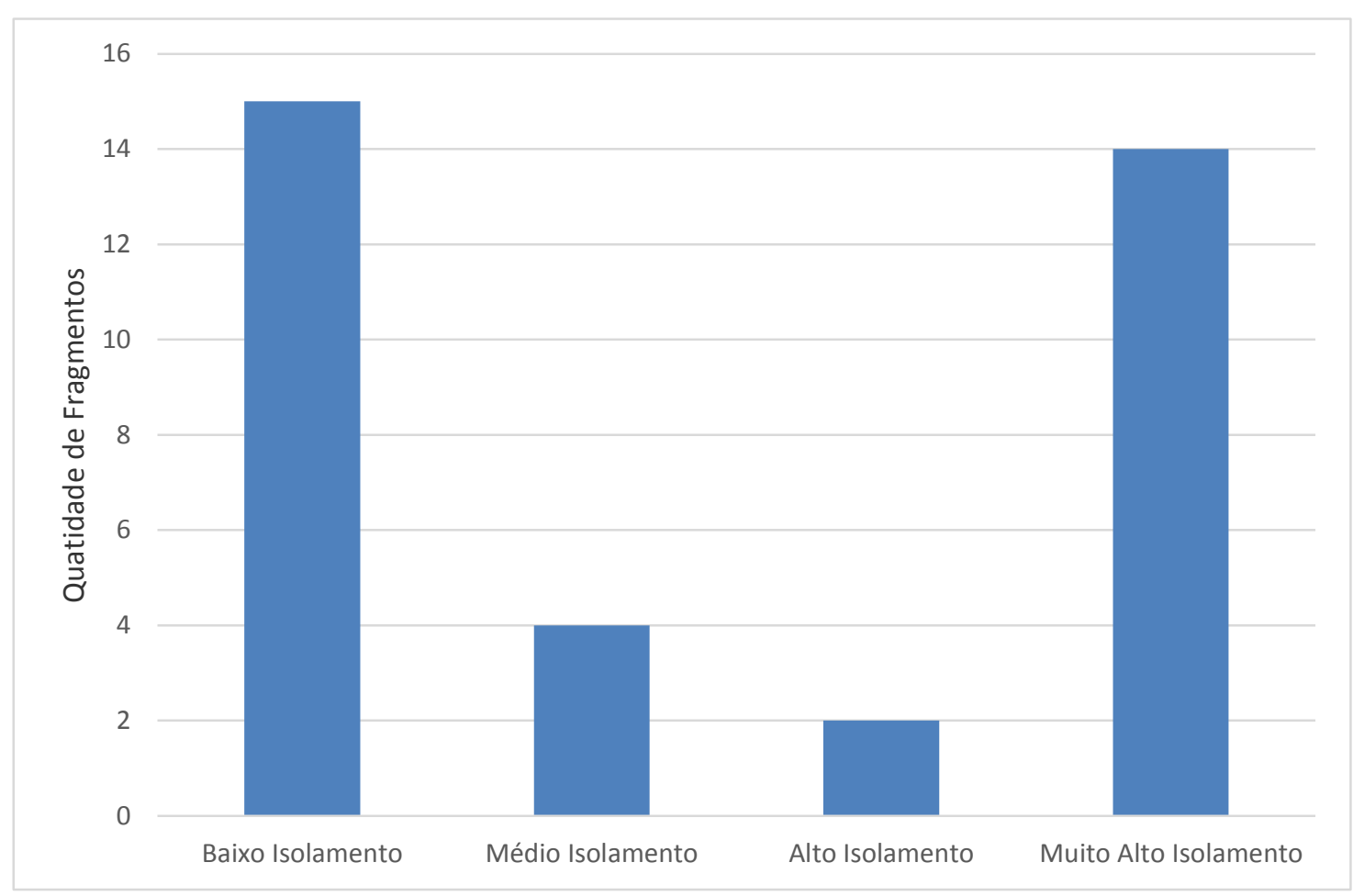

Figura 3. Quantidade de fragmentos com baixo, médio, alto e muito alto isolamento. Fonte: Dados da Pesquisa.

Com a análise dados foi possível observar grupos de fragmentos muito próximos, sendo eles os fragmentos: 1, 2, 3, 4 e 7, que apresentam entre si, uma distância abaixo de 60 metros, caracterizando um baixo isolamento, o que demostra grande potencial para implantação corredores ecológicos (Figura 4).

Outros fragmentos muito próximos entre si, são: 8, 9, 11, 12 e 27, onde aqueles de numeração 11 e 27 parecem desempenhar a função de um corredor entre os fragmentos 8 e 12 . O mesmo parece ocorrer entre os fragmentos: 5, 28, 29 e 30, e também entre aqueles identificados pelos números: 32, 33 e 34, todos localizados na região oeste da bacia. Ainda nessa mesma região, destaca-se o fragmento 31, por apresentar uma área de 33,88 ha, sendo o maior fragmento da bacia. O mesmo tem um formato irregular e um grau de isolamento médio, tendo como vizinho mais próximo o fragmento 12 . O fragmento 31 precisa ser preservado e conectado ao seu vizinho mais próximo, pois por ser o maior fragmento da bacia do alto curso do rio Gramame, apresenta um grande potencial para abrigar espécies.

No lado leste da bacia existe menor número de fragmentos em relação à região oeste. Além disso, os mesmos apresentam uma área total menor que a área dos fragmentos da região oeste, mostrando que essa porção da bacia é mais preservada. 


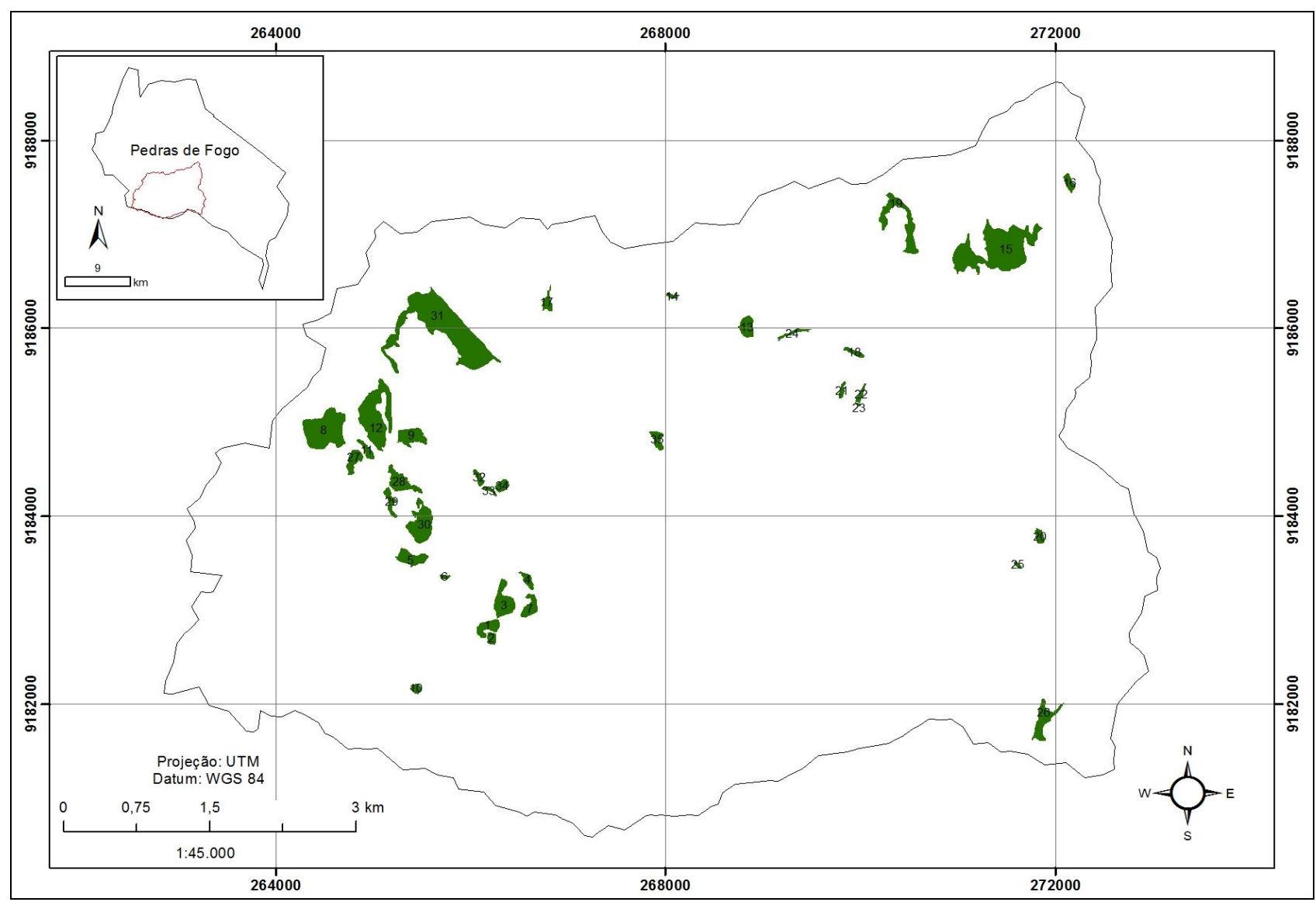

Figura 4. Mapa dos fragmentos florestais existentes na bacia do alto curso do rio Gramame, município de Pedras de Fogo (PB). Fonte: Dados da Pesquisa.

\section{CONSIDERAÇÕES FINAIS}

Os fragmentos da bacia do alto curso do rio Gramame apresentam uma área muito reduzida devido à degradação ocorrida para dar lugar ao plantio de cana-de-açúcar. Essas reduções alteraram as condições físicas no interior dos fragmentos, caracterizando efeito de borda, tendo consequências negativas para a vegetação, causando maior mortalidade das plantas, como também alterando o comportamento das espécies que usam a floresta como abrigo e refú gio. Certamente a fragmentação ocorrida ao longo dos anos tem acarretado mudanças significativas na dinâmica local das espécies, pois as alterações das condições físicas no interior dos fragmentos determinam quais espécies podem tolerá-las sem que sejam forçadas a migrar.

Quanto à preservação dos fragmentos, observou-se que os mais recomendados foram os que estão na região oeste da bacia, onde é possível observar a existência de grupos muito próximos que podem ser interligados, aumentando assim a área disponível para as espécies e também diminuído os efeitos de borda.

Como próximo passo, torna-se necessária a realização de um estudo detalhado sobre as espécies existentes na bacia, para que, junto com os dados das análises dos fragmentos feitas neste 
trabalho, sejam traçadas metas eficientes para a preservação, levando em consideração as formas de dispersão de cada espécie.

\section{REFERÊNCIAS}

ALMEIDA, C. G. Análise espacial dos fragmentos florestais na área do parque nacional dos capôs gerais, Paraná. 72f. 2008. Disertação (Mestrado em Gestão do Território) - Universidade Estadual de Ponta Grossa, Ponta Grossa, 2008.

BARbosa, J. E. L.; WATANABE, T.; PAZ, R. J. A Bacia do Rio Gramame: biodiversidade, uso e conservação. Campina Grande: EDUEPB, 2009.

BRASIL. Ministério do Meio Ambiente. Fragmentação de ecossistemas: causas, efeitos sobre a biodiversidade e recomendações de políticas públicas. Brasília: MMA/SBF, 2003.

BRASIL. Ministério de Minas e Energia. Diagnóstico do Município de Pedras de Fogo. Recife, 2005.

GUARIZ, H. R.; CAMPANHARO, W. A.; PICOLI, M. H. S. Avaliação do tamanho e forma de fragmentos florestais por meio de métricas de paisagem. Congresso Brasileiro de Reflorestamento Ambiental; 2011 Set 14-16; Guarapari, ES; 2011.

ODUM, E. P. Fundamentos de Ecologia. 6ª ed. Lisboa: Fundação Calou sete Gulbenkian, 2004. LIMA, J. C.; FURIER, M.; GUEDES, E. C. R. Áreas de Preservação Permanente ocupadas com cana-de-açúcar: o caso das encostas íngremes em Itambé/PE e Pedras de Fogo/PB. Okara: Geografia em Debate, v. 5, n. 1-2, p. 46-60, 2011.

MELO, A.S.T.; HECKENDORFF, W.D.; ALVES, E.L.; GUIMARÃES, M.M.M. O meio ambiente natural: componentes abióticos e bióticos. In: MELO, A.S.T. et al. (Org.). Projeto de Pesquisa: Vale do Jaguaribe. João Pessoa: Ed. UNIPÊ, 2001.

PEREIRA, M.; ALVES, R. Composição florística de um remanescente de Mata Atlântica na Área de Proteção Ambiental Barra do Rio Mamanguape, Paraíba, Brasil. Revista de Biologia e Ciências da Terra, v. 7, n. 1, p. 1-10, 2007.

RICKLEFS, R. E. A Economia da Natureza. 6 ed. Rio de Janeiro: Guanabara Koogan, 2010.

SILVA, M. S. F.; SOUZA, R. M. Padrões espaciais de fragmentação florestal na Flona do IburaSergipe. Mercartor, v. 13, n. 3, p. 121-137, 2014.

TOREZAN, J. M. D. Fragmentação Florestal e Prioridades para conservação da Biodiversidade. 2003. Disertação (Mestrado em Engenharia Ambiental) - Universidade de São Paulo, São Carlos, 2003.

TOWNSEND, C. R.; BEGON, M.; HARPER, J. Fundamentos em Ecologia. 3 ed. Porto Alegre: Artmed, 2010.

Recebido em: 14/08/2016

Aceito para publicação em: 01/10/2016 